\title{
Myelin growth and coiling
}

\author{
Mohit Haran, Ashim Chowdhury, C. Manohar, Jayesh Bellare*
}

Department of Chemical Engineering, IIT Bombay, Mumbai 400 076, India

\begin{abstract}
Surfactants form a variety of microstructures in solution, some of which are equilibrium and the others are non-equilibrium microstructures. During the dissolution of a surfactant in a solvent, various types of non-equilibrium microstructures have been observed. The most interesting and fascinating non-equilibrium microstructure is the 'Myelin Figure'. In the present report the swelling and dissolution dynamics of the myelin growth in the surfactants Aerosol-OT (AOT) and phosphatidylcholine (PC) in aqueous medium have been studied. The effect of the additive para-toluene sulfonic acid (PTS) on the dynamics and the shape of these myelin structures have been investigated. The observation of coiling transformations in the presence of these additives has been studied. It is shown that the molecular diffusion of the surfactant from the interface and the flexibility of the interface are the two important parameters for formation, growth and coiling of myelin structures. Chemical reactions in myelins have been carried out to throw light on the water transport during the myelin growth.
\end{abstract}

Keywords: Myelins; Coiling; Aerosol-OT; para-Toluene sulfonic acid; Phosphatidycholine

\section{Introduction}

Surfactants are the basic components of most commercial cleaning products. Thus, an understanding and control of their dissolution behavior is important for enhancing their applications and performance. This necessitates the study of dynamic microstructures formed as they progress towards equilibrium. Lamellar phases of surfactants like Aerosol-OT (AOT) and phosphatidylcholine (PC), when contacted with excess water show the growth of one such non-equilibrium
microstructure-'Myelins', which were first observed in lipids by Virchow [1]. Myelins are highly viscous, gel-like, microstructures consisting of multilamellar tubules of alternating amphiphile bilayer and water layer with core axis of water (Fig. 1).

Myelin growth is a manifestation of the dynamic interface instability involving the rearrangement of bilayers of the lamellar phase of a nearly insoluble surfactant in the excess of solvent. Buchanan et al. [2] suggested that the nearly immiscible fully swollen lamellar phase of surfactant takes the form of myelins because of the aggregative diffusion of surfactant towards the solvent to establish equilibrium with the inward diffusion of the solvent. 
In this paper, we investigate the dynamics of myelins formed by AOT and PC in aqueous medium, with controlled addition of additives like para-toluene sulfonic acid (PTS). This molecule was chosen because PTS acts as a bilayer-penetrating agent and we expected that its addition would affect the myelin formation and growth. To our surprise, we observed not only the effect on growth but also its effect on the coiling of myelins. These studies have been carried out using video and SEM microscopy. For microscopic observations, procedures given in the literature [5] are followed. Some of our observation like a marked increase in coiling is similar to the reports of Frette et al. [4] who used a polymeric additive while this work uses a much smaller molecule. They have proposed a model where they assume that the polymer modifies the spontaneous curvature locally and have argued that a critical surface concentration of the colloidal additives is necessary to induce coiling. Further, chemical reactions in lamellar phase doped with one reactant and the aqueous phase with the other reactant were carried out to understand the growth of myelins and the diffusion mechanism of water.

Sahkurai and Kawamura [5] have studied the myelin growth in egg-yolk phosphatidycholine. They found the process to be diffusion-like with the effective diffusion coefficient $D$ of approximately $10^{-6} \mathrm{~cm}^{2} \mathrm{~s}^{-1}$. Mishima and Yoshima [6] observed the same thing in the PC/water system. Buchanan et al. [3] have studied the myelin growth in non-ionic surfactants with large miscibility gap (viz. $\mathrm{C}_{12} \mathrm{E}_{3}$ ) with water.

\section{Materials}

Sodium bis(2-ethylhexyl)sulfosuccinate or AOT (purity 99\%) and L-2 Phosphatidycholine (type iv-s: from soyabean of purity $40 \%$ ) from Sigma Chemical Co., USA were used as surfactants. PTS from the Spectrochem Pvt Ltd, India (purity $98.5 \%$ ) was used as the bilayer-penetrating agent. Chloroform (purity 99.5\%) and methanol (purity 99.8\%), both from Sisco Research Laboratory Pvt Ltd, India were used as casting solvents. Calcium nitrate (purity 98\%) from Merck Ltd (India) and anhydrous Sodium carbonate (purity 99.9\%) from Qualigens Fine Chemical, India were used for the reaction study. All these chemicals were used without further purification.

\section{Experiments}

\subsection{Optical microscopy}

All observations were made using the OLYMPUS BX60 type microscope in transmitted and polarized light mode. The images were recorded using a CCD camera (Sony XC-77CE model) together with a frame-grabber PC. The micrographs were captured and analyzed using the image processing software, IMAGE PRO-PLUS.

Samples for the phase penetration studies were prepared by drying 2-3 drops of surfactant dissolved in the chloroform-methanol solution between the glass slide and the coverslip. The lump
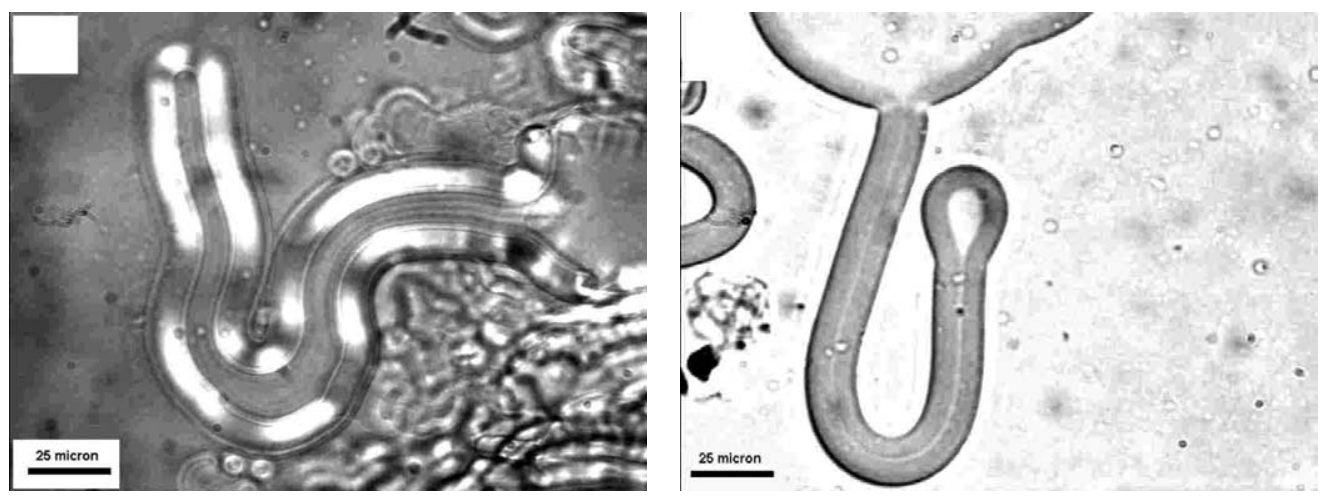

Fig. 1. Myelin description. 

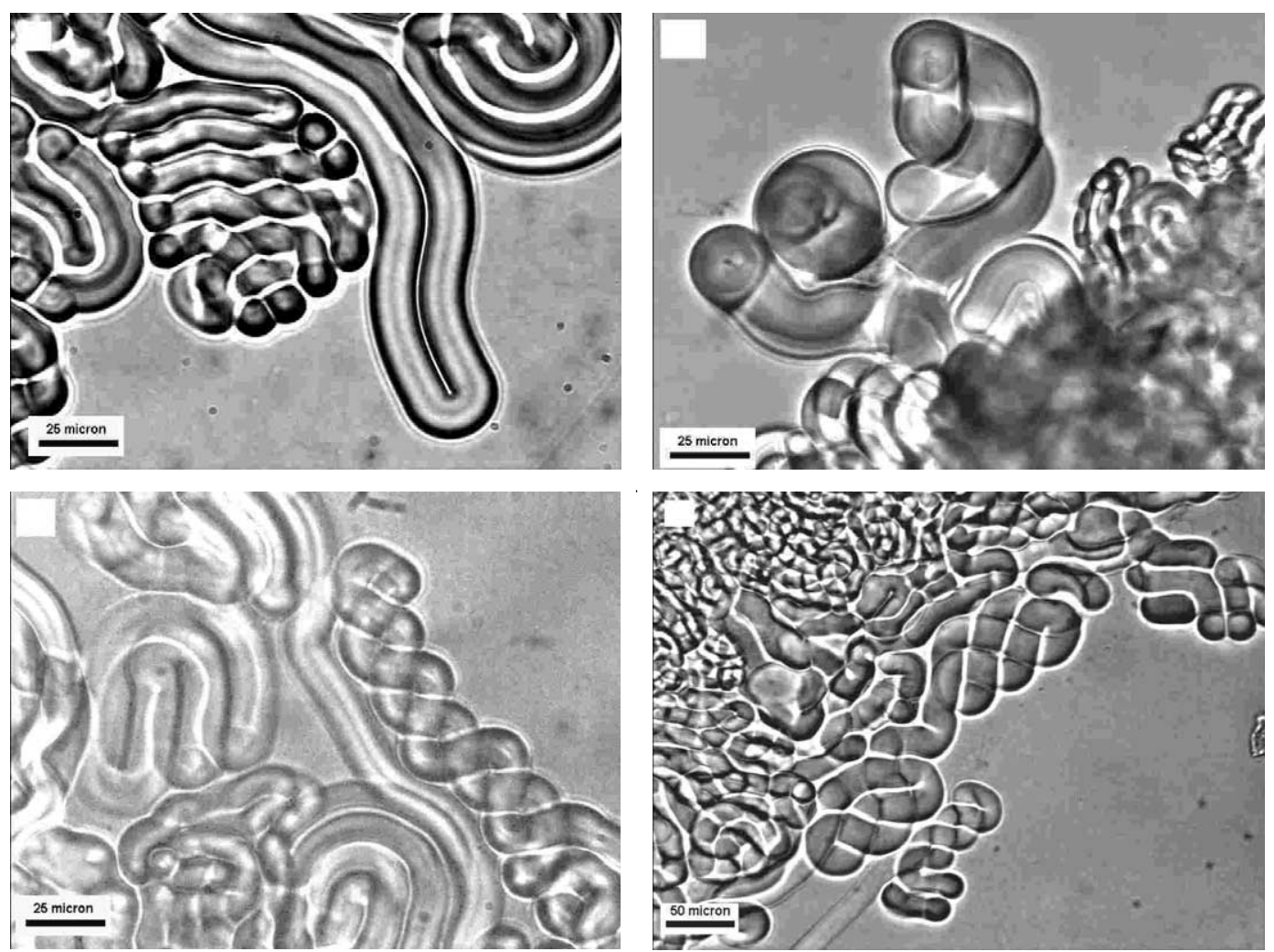

Fig. 2. Micrographs showing various microstructures.

formed was pressed slightly under the coverslip and contacted with 1-2 drops of dispersive liquid at the edge of the coverslip. The dynamics of the microstructures formed was followed using optical microscope. All experiments were carried out at room temperature.

\subsection{Scanning electron microscopy}

Scanning electron microscopy was used to study the surface topography of the non-equilibrium microstructures formed. All observations were made using the JSM-6400 model of JEOL, Japan under cryogenic conditions of liquid $\mathrm{N}_{2}$ [7].

In sample preparation, a drop of surfactant lump was taken in a hole of clean and dry stub to which 2-3 drops of water was added. Myelin growth was then vitrified by rapidly plunging it into a pool of liquid Freon using spring-loaded plunging device [8]. After $5 \mathrm{~min}$ of temperature stabilization, the stub was transferred to liquid $\mathrm{N}_{2}$ and shifted rapidly to the previewing stage. It is then cryo-fractured using an internal knife and evaporative gold coated to view the cross-section under SEI mode [9].

\section{Results and discussion}

Based on the preliminary experiments AOT and PC were chosen as surfactants for the study because both are known to show myelin structures and are amenable for growth rate measurements. These studies established the conditions for observing the myelin growth. Other molecules like SDS and CTAB did not show the myelin growth. 

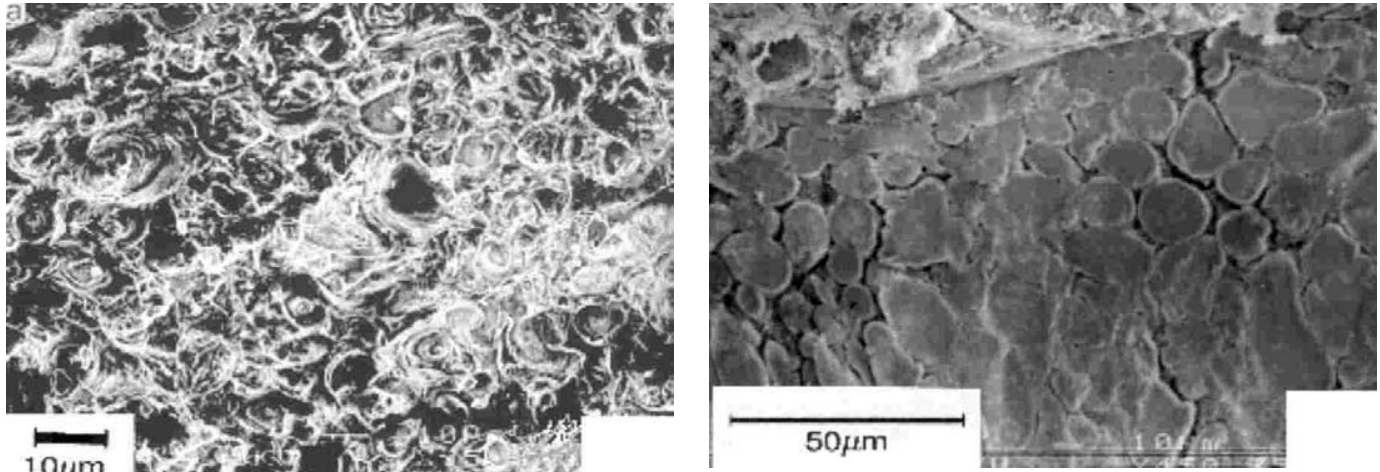

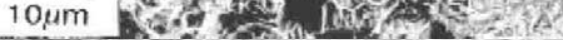
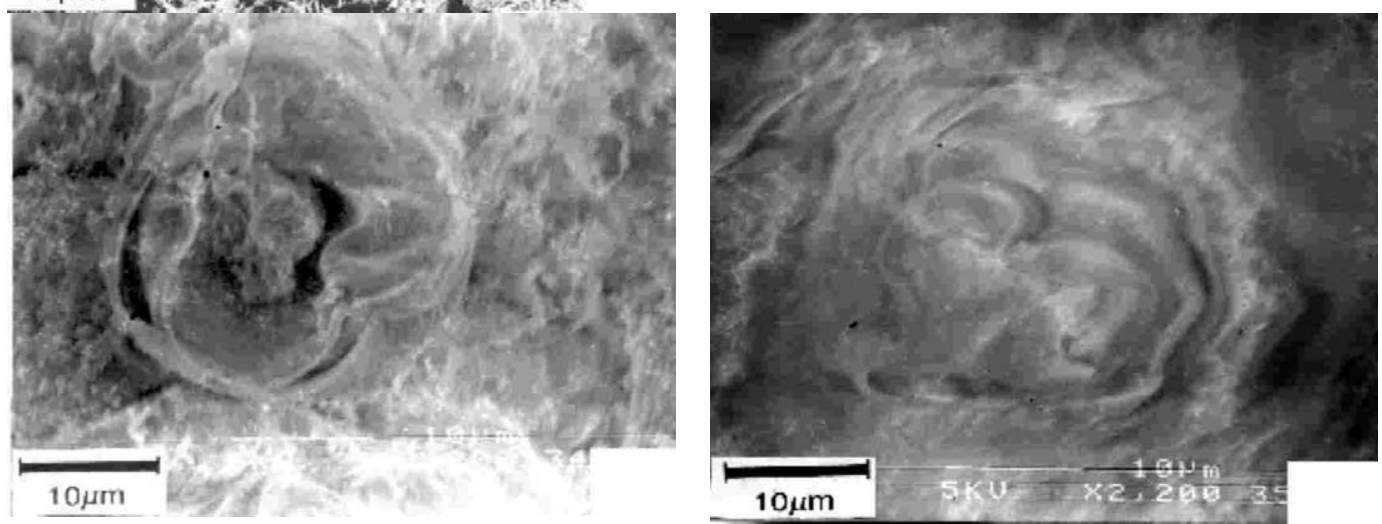

Fig. 3. Cryo-SEM of PC myelins showing their lamellar structure.
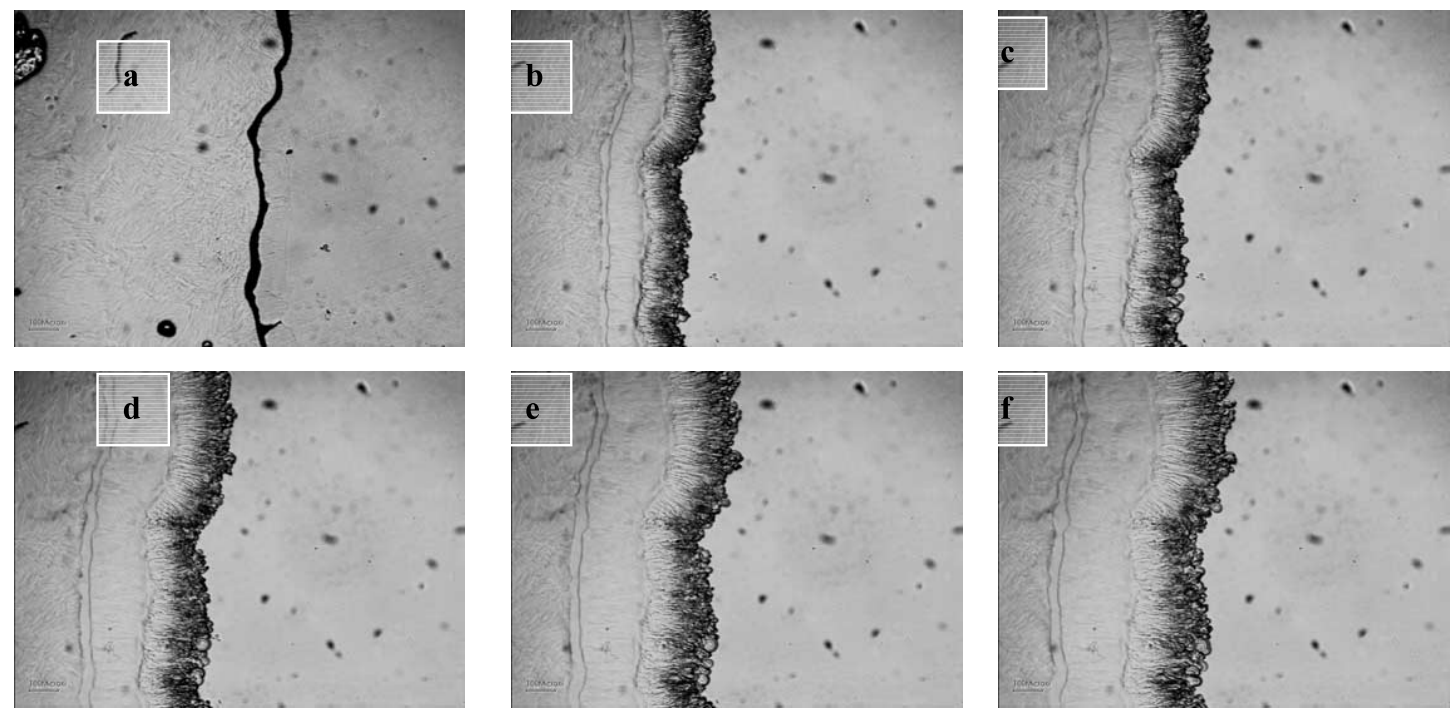

Fig. 4. Time series micrographs of myelin growth in $\mathrm{PC} /$ water system. 


\section{PC/ Water System}

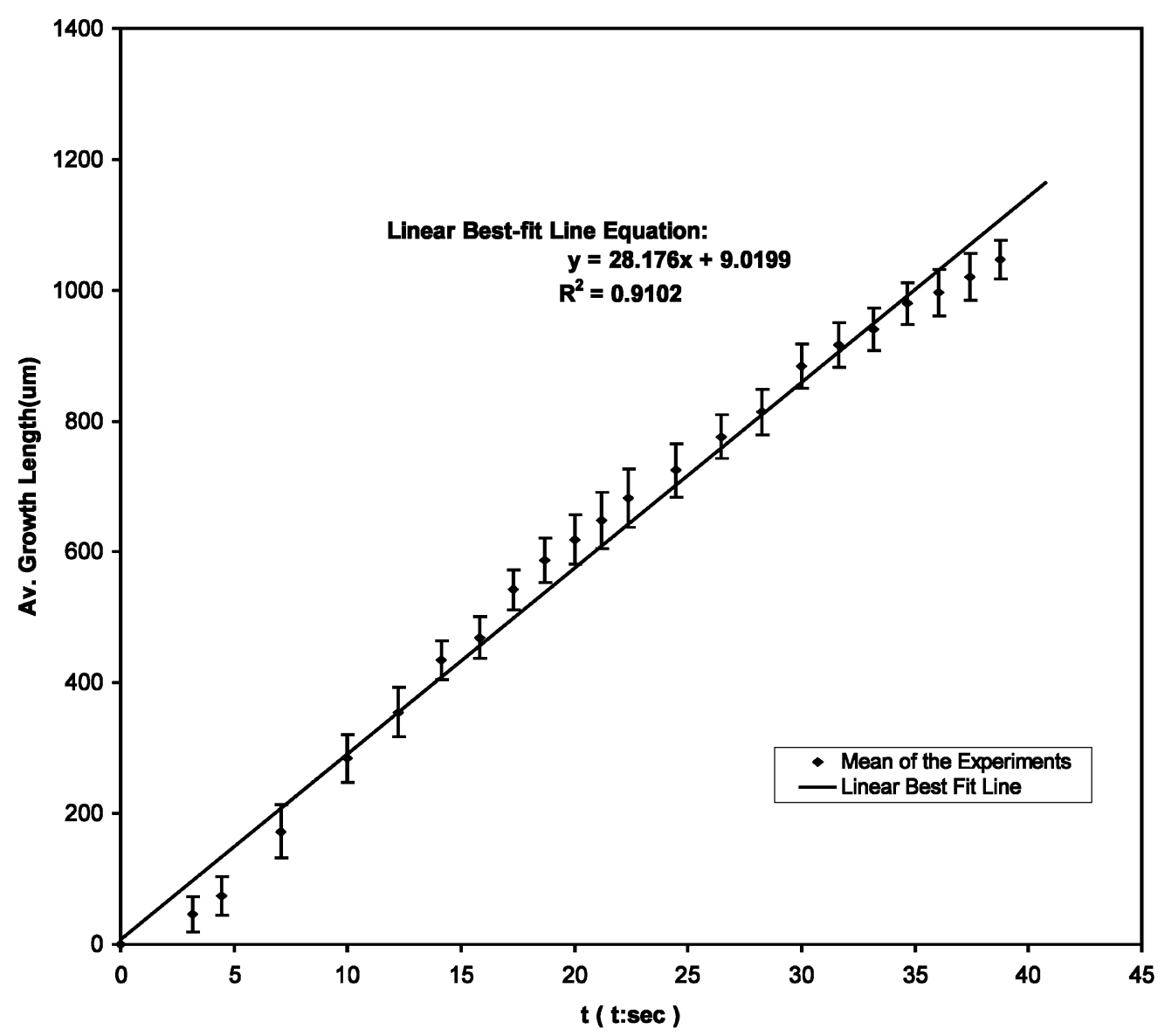

Fig. 5. Plot of $L^{2}$ vs. $t$ for PC, to calculate the effective diffusion coefficient.

Table 1

Effective diffusion values

\begin{tabular}{lllll}
\hline Sr. no. & Surfactant & Liquid medium & Best fit line equation & $D \times 10^{6}\left(\mathrm{~cm}^{2} \mathrm{~s}^{-1}\right)$ \\
\hline 1 & PC & Water & $y=28.176 x+9.019$ & 3.96 \\
2 & PC & Ethylene glycol & $y=9.6004 x+31.115$ & 0.46 \\
\hline
\end{tabular}

\subsection{Phosphatidylchloine system}

The time series evolution of the lamellar/liquid interface on the contact of water with $\mathrm{PC}$ was observed. After an initial delay, simple rod-like myelins with an average diameter of $10-30 \mu \mathrm{m}$ and almost perpendicular to the interface were observed. Under polarized light, these appeared in three phases, with the myelin phase appearing bright. After 3-5 min, complex structures like double helices, tadpoles and vesicles are formed (Fig. 2), which kept for longer times fused together. On flooding the surfactant with water by using a concave slide without the coverslip, worm- 


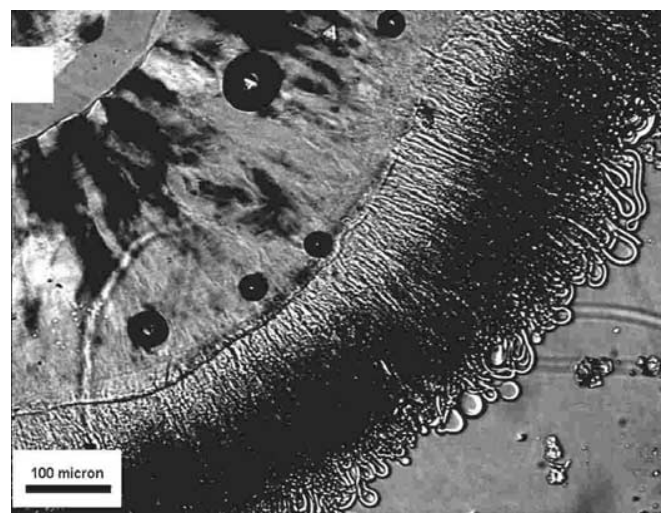

Fig. 6. Phase differentiation under polarized light.

like structures, especially vesicles at the top of myelin tails are formed. Cryo-SEM on the lateral fracture of the cryo-fixed myelins showed a clear multilamellar texture of the myelin cross-structure which was visible (Fig. 3).

From a series of micrographs as shown in (Fig. 4) the myelin growth was quantitatively analyzed by measuring the distance of the myelin front from the root as a function of time $(t)$ from the beginning of growth of all the systems examined. The mean of average growth length $(L)$ of different sets is plotted against the square root of the corresponding time and a best-fit straight line is obtained for short periods (Fig. 5), suggesting the diffusion process as the rate-limiting process in accordance to the earlier work [5]. Theoretically, the mean distance $(L)$ translated in a diffusional process during time is given by

$L^{2} \approx C+k D t$

where $D$ can be considered as an effective diffusion coefficient. Thus from the slope of the best fit line apparent diffusion coefficient for the PC/water and PC/ethyleneglycol systems was calculated as shown in Table 1.

The transport of PC molecules in the form of myelin aggregates maybe attributed to their amphiphilic nature, which inhibits their dissolution molecularly into the medium. During growth, thickening of myelins in radial direction is hampered by elastic constraints. Further, radial diffusion coefficient has been reported to be much lower than the lateral diffusion coefficient.
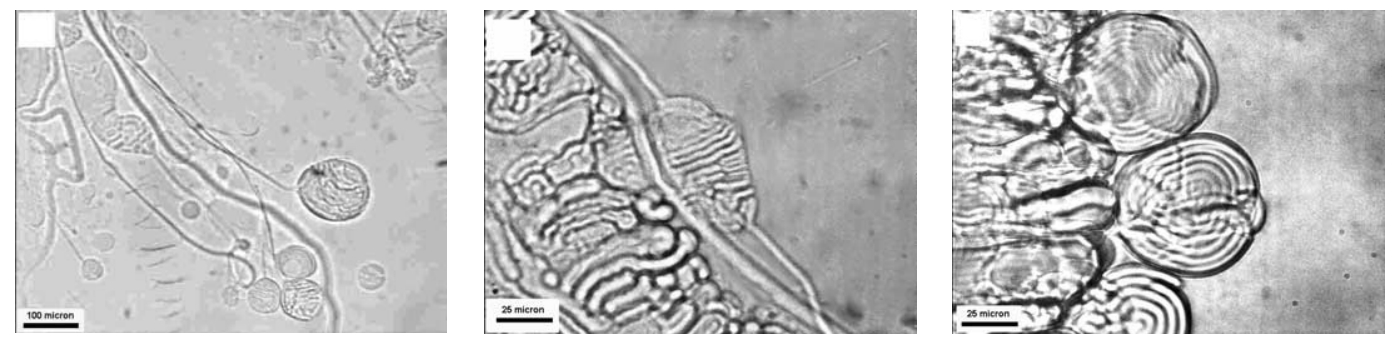

Fig. 7. Magnified micrograph of vesicles to show the coiled myelins.

Table 2

AOT/water system

\begin{tabular}{lllll}
\hline Sr. no. & Surfactant & Solvent & Best-fit line equation ${ }^{\mathrm{a}}$ & $\mathrm{D} \times 10^{6}\left(\mathrm{~cm}^{2} \mathrm{~s}^{-1}\right)$ \\
\hline 1 & AOT & Water & $y=19.363 x-20.58$ & 1.87 \\
2 & AOT & $0.2 \mathrm{wt} \%$ AOT solution (aq.) & $y=18.517 x-28.843$ & 1.71 \\
3 & AOT & $0.5 \mathrm{wt} \%$ AOT solution (aq.) & $y=14.688 x-11.903$ & 1.08 \\
4 & AOT & $1.0 \mathrm{wt} \%$ AOT solution (aq.) & $y=12.855 x-27.649$ & 0.83 \\
5 & AOT & $1.5 \mathrm{wt} \%$ AOT solution (aq.) & $y=11.875 x-31.203$ & 0.78 \\
\hline
\end{tabular}

a $y=$ mean of the average growth length in $\mu \mathrm{m}, x=\sqrt{ }$ time in s. 

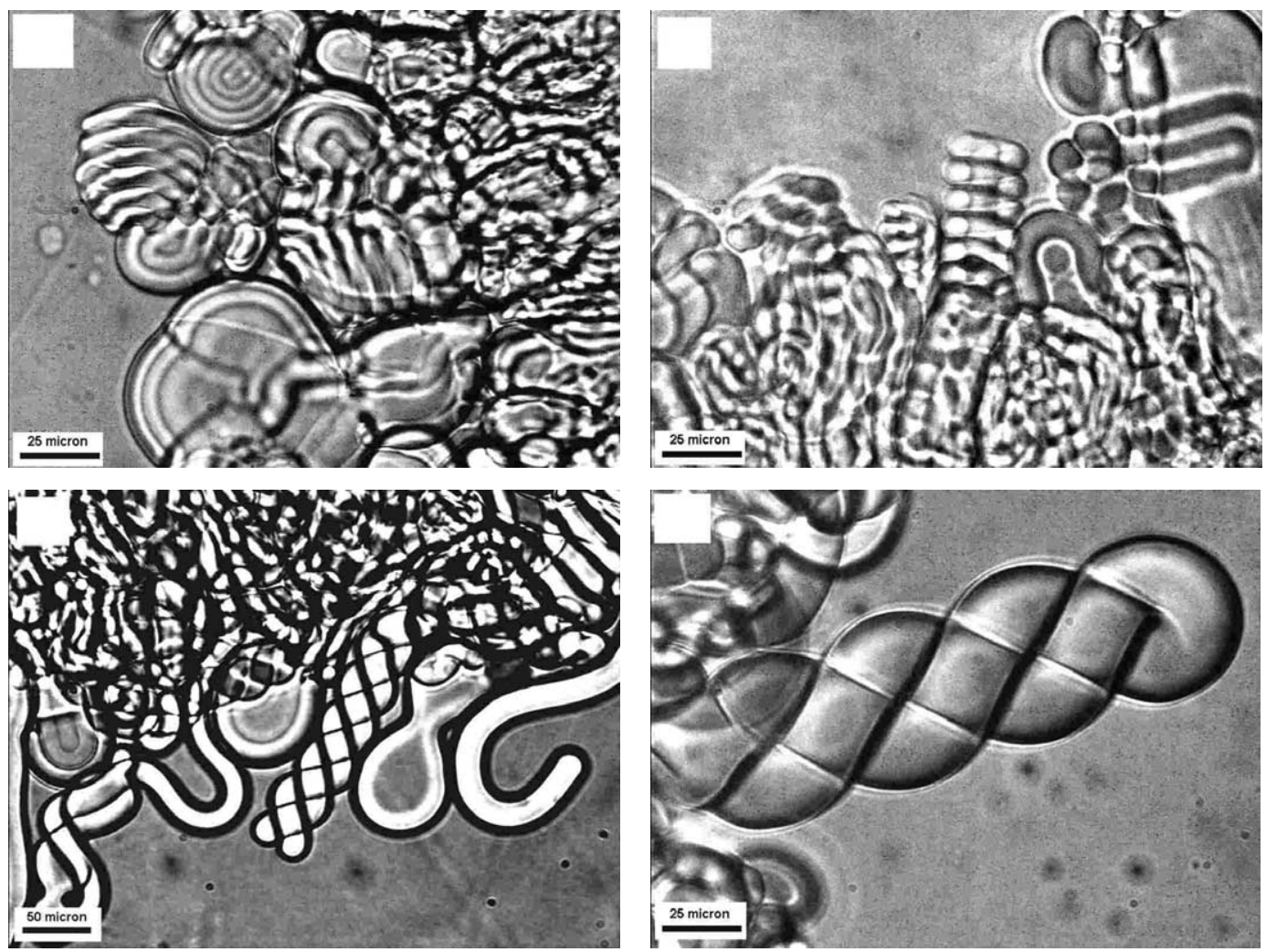

Fig. 8. Coiling instabilities in AOT $/ 5 \mathrm{wt} \%$ aq. PTS solution.

Table 3

Radius to diameter ratio for double helix

Average diameter of the myelin rods forming double helix $(\mu \mathrm{m})$

29.8

19.5

10.2

15.5

14.8

10.7
Average pitch of the double helix $(\mu \mathrm{m})$

$\begin{array}{ll}46.9 & 0.64 \\ 25.4 & 0.77 \\ 13.1 & 0.78 \\ 23.4 & 0.66 \\ 21.8 & 0.67 \\ 17.9 & 0.61\end{array}$

\subsection{Aerosol-OT}

On contact with water, AOT also forms dynamic microstructures like $\mathrm{PC}$, but unlike $\mathrm{PC}$ it does not show them on contact with ethylene glycol. The myelin figures observed had average radii of $10-15 \mu \mathrm{m}$. Distinctly, under polarized light, AOT system formed five different phases as against the three observed in the PC system (Fig. 6). Phase 1 is the surfactant lamellar phase, phase 4 is the myelin phase and phase 5 is the solvent phase. Besides these three phases observed in PC, two new phases (2 and 3) were also observed. Under polarized light, phase 2 appeared dark, implying a non-liquid crystalline phase unlike other phases. At longer times, vesicle-like struc- 
Table 4

Tubular radii of coils

\begin{tabular}{lll} 
Figure number & $\begin{array}{l}\text { Tubular radii of } \\
\text { single/double helix } \\
(\mu \mathrm{m})\end{array}$ & $\begin{array}{l}\text { Tubular radii of } \\
\text { uncoiled myelin } \\
(\mu \mathrm{m})\end{array}$ \\
\hline (a) & 4.6 & 7.5 \\
(b) & 5.6 & 8.0 \\
(c) & 5.7 & 7.1
\end{tabular}

tures are more dominant with less of coils and helices being formed. Vesicle-like structures are formed by the extensive coiling of myelins, as observed by the magnified micrographs of the vesicles in Fig. 7.

The growth rate of myelins in AOT was studied using time series micrographs and the apparent diffusion coefficient was calculated (Table 2). We argued that if the myelin formation is triggered and controlled by the AOT molecules from the lamellar phase diffusing into the water phase, then reducing the concentration gradients of AOT should slow down the growth rate of myelins. This gradient control was achieved by taking dif- ferent concentrations of AOT in the water phase that is brought into contact with the lamellar phase. These experiments showed that there was no observable change in the structural features of the myelin figures that were formed. However, there was a reduction in the growth rate with an increase in the AOT concentration (Table 2). This decrease was consistent with the picture of AOT diffusing from the surface of the membrane, enhancing the Helfrich fluctuations [12].

\subsection{Bilayer penetrating agent: PTS}

The second aspect, we wanted to check, was the role of the rigidity of the lamellar membranes in myelin formation. PTS, which is known to be a hydrotrope, consequently acts as the membranepenetrating agent, should be ideally suited for modifying rigidity.

Studies with PTS were carried out in two ways: (a) by taking the PTS water phase; and (b) PTS in the surfactant phase. The study of myelin growth in the presence of the bilayer-penetrating agent held some surprises. In the first case, the growth
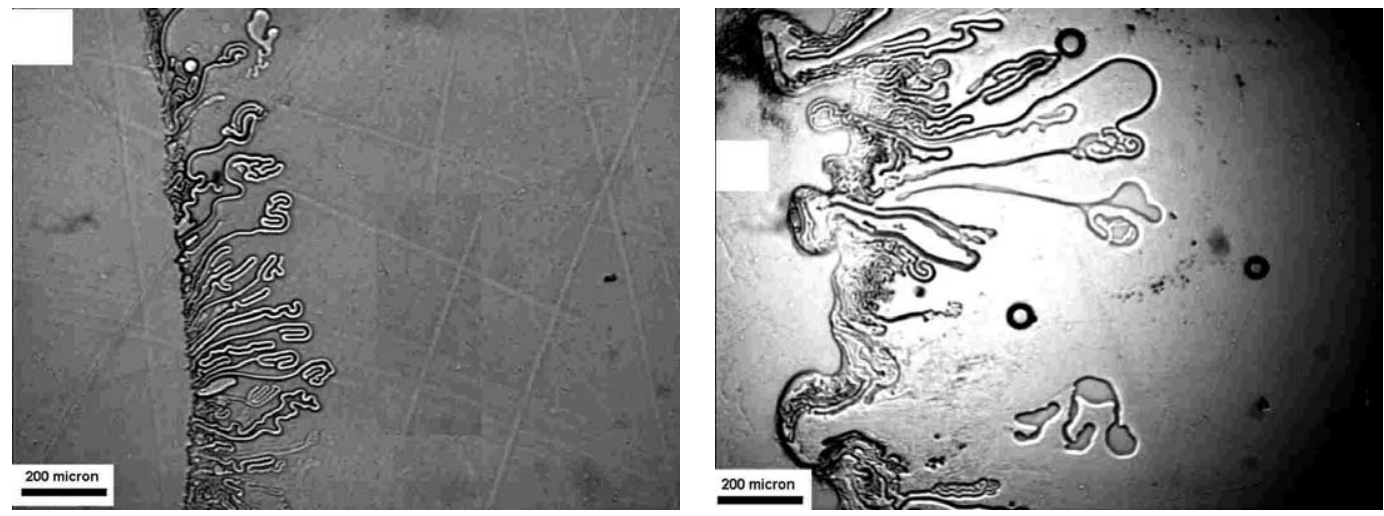

Fig. 9. PTS in lamellar phase, with rupture of lamellar phase.

Table 5

Summary of results of growth rate study in AOT systems with PTS solution (aq.) as the liquid medium

\begin{tabular}{lllcl}
\hline Sr. no. & Surfactant & Solvent & Best-fit line equation ${ }^{\text {a }}$ & $D \times 10^{6}\left(\mathrm{~cm}^{2} \mathrm{~s}^{-1}\right)$ \\
\hline 1 & AOT & $1 \mathrm{wt} \%$ PTS solution (aq.) & $y=9.8857 x+16.237$ & 0.48 \\
2 & AOT & $5 \mathrm{wt}^{2} \%$ PTS solution (aq.) & $y=11.017 x-4.864$ & 0.61 \\
\hline
\end{tabular}

\footnotetext{
a $y=$ mean of the average growth length in $\mu \mathrm{m}, x=\sqrt{ }$ time in s.
} 

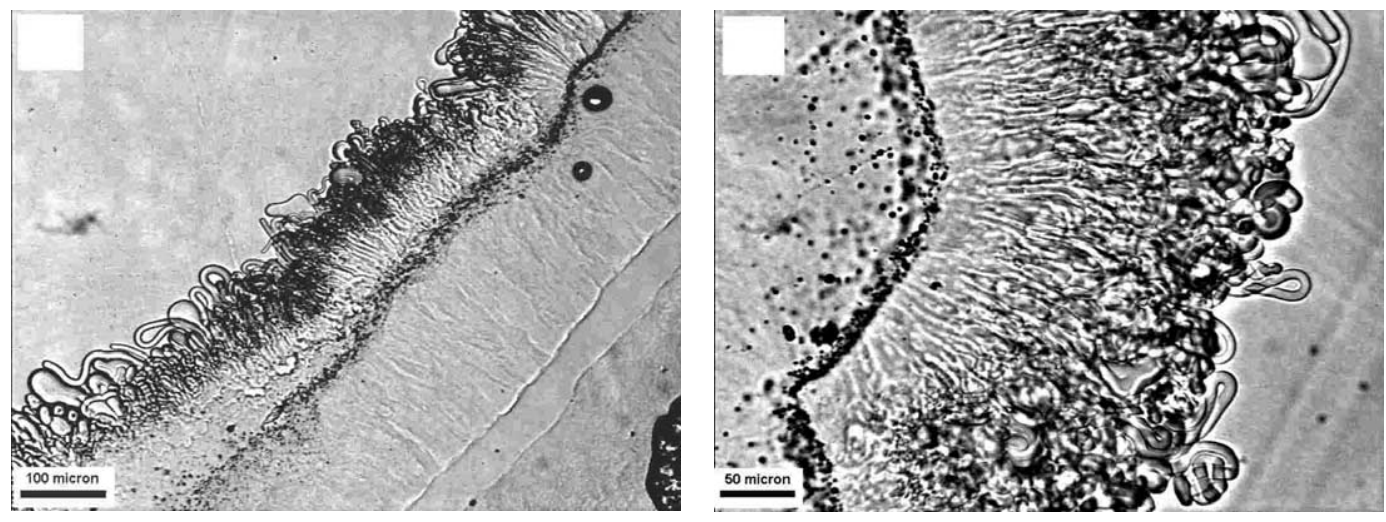

Fig. 10. Chemical reaction in lamellar phase with precipitate in lamellar phase.

rate was affected as anticipated. However, the nature of myelin was drastically changed by the spectacular formation coiling instabilities. In AOT/ pure water system, during short times straight rod-like myelins are the dominant microstructures. However, using the aqueous solution of PTS as the liquid medium, with the PTS concentration above $1 \mathrm{wt} \%$, a large number of complex structures like coils and helices start forming. Fig. 8 shows the myelin structures in $\mathrm{AOT} /(5 \mathrm{wt} \%)$ aq. PTS solution system. Initially a large number of looped rods (diameter ranging between 9 and $12 \mu \mathrm{m}$ ) were observed which at later stages formed single and double helices, tadpoles, etc. Single helices observed had pitches almost equal to the rod diameter. It appeared that the formation of new instabilities required a threshold concentration of about $1 \%$.

Frette et al. [3] have used hydrophilic dextran polymer as the bilayer anchoring agent to study the coiling instability in PC and have concluded that the polymer added changes the spontaneous curvature in the membrane. They have, using a model, argued that coiling instabilities are formed beyond a critical concentration. Lipowsky and Heirgiest [10] have studied the effect of polymeric anchoring agent on the elastic properties of the bilayers, which affect the quasi-equilibrium shape formed during growth.

Two different types of double helices were observed to be formed - one by the coiling of two different myelin rods and the other formed by the coiling of a single rod, with their ratio of rod diameter to pitch lying in a short range as given in
Table 3. Averaging the diameter of various instabilities, it was found that the straight rod had the maximum diameter, followed by looped rods and finally double helices, suggesting that coiled structures are preferred by smaller diameter rods [11] (Table 4). With $10 \mathrm{wt} \%$ PTS solution, significant increase in coiling in short times was observed. Significantly the ratio of rod diameter to pitch of the helices remained within the range of $0.6-0.8$. Further, the growth rate was found to be much lower than that for the pure water system, with a lower effective diffusivity coefficient.

The effect of PTS in lamellar phase was also studied by incorporating the PTS in the AOT lamellar phase. Three different AOT-PTS solutions with PTS concentrations of $0.8,1.0$ and $1.5 \mathrm{wt} \%$ in

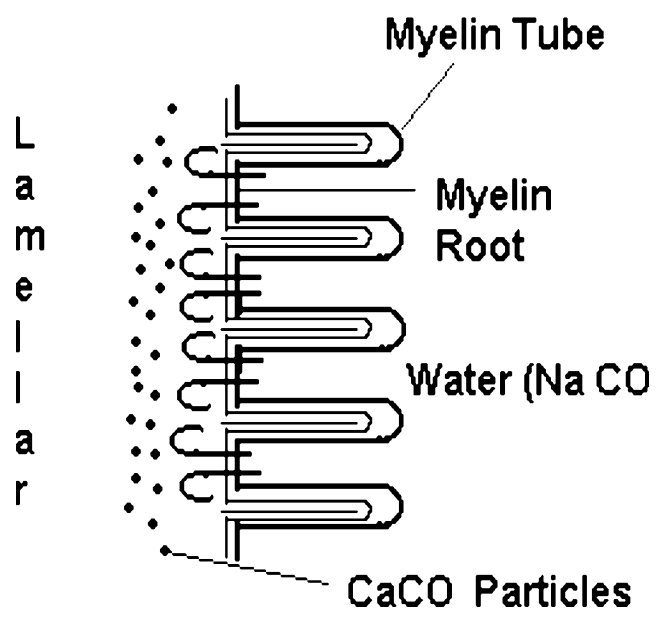

Fig. 11. Myelin growth model. 
$10 \mathrm{wt}^{\%} \%$ AOT each were used. For $1 \mathrm{wt} \%$ PTS solution, water penetrates into the lamellar phase after 3-4 min of contact by rupturing it (Fig. 9), while in case of $1.5 \mathrm{wt} \%$ it penetrates as soon as the contact is made. Myelin figures are formed in lower concentrations of $0.8 \mathrm{wt} \%$, but the texture is different from pure AOT systems. Myelins are quite wide apart from the very beginning of their growth, even at the root zone, and no complex structures like double helices are formed. This suggests a loosening of the lamellar structure due to the uniform distribution of PTS in lamellar phase (Table 5).

\section{Chemical reaction}

To get a better insight of the myelin growth mechanism, precipitation chemical reactions in the lamellar phase during myelin formation have been studied. The reaction used is the formation of insoluble $\mathrm{CaCO}_{3}$ particles via the following reaction:

$\mathrm{Ca}\left(\mathrm{NO}_{3}\right)+\mathrm{Na}_{2} \mathrm{CO}_{3} \rightarrow \mathrm{CaCO}_{3} \downarrow+2 \mathrm{NaNO}_{3}$

A uniform dispersion of chloroform solution of AOT and $0.1 \mathrm{M} \mathrm{Ca}\left(\mathrm{NO}_{3}\right)_{2}$ solution was used as the lamellar phase with $0.1 \mathrm{M} \mathrm{Na}_{2} \mathrm{CO}_{3}$ as the liquid medium. After 1-2 min of contact, insoluble $\mathrm{CaCO}_{3}$ particles precipitated in a very small region near the root (Fig. 10). The same phenomenon has been observed by Hota et al. [13] using the $\mathrm{CaCl}_{2}$ solution. This observation clearly suggests that water does not penetrate deep into the lamellar phase, but takes a 'U' turn to get inside the myelin rods after diffusing into the bilayer membrane. (Fig. 11).

\section{Conclusion}

Present studies on myelin growth in PC and AOT systems show the following:

(A) Molecular diffusion of the surfactant from the surface of the lamellar phase plays an important role in the growth of myelins. Reducing the concentration gradients decreases the growth rates of myelins.
(B) PTS incorporated in to penetrating water phase modifies the interface drastically and generates, beyond a threshold concentration, coiling in myelins. This points out that the flexibility of the bilayer is an important parameter contributing to the structure and dynamics of myelin and other non-equilibrium structures.

(C) Drastic changes occur in myelin formation when PTS is incorporated in to the surfactant phase with the virtual disappearance of myelin-like structures.

(D) Formation and deposition of $\mathrm{CaCO}_{3}$ particles indicates organized water flow on the length scales of several hundred microns.

\section{Acknowledgements}

We thank Dr. Peter Garrett, Dr. Vinod Dhanuka, and Dr. Satish Goel for discussions.

\section{References}

[1] R. Virchow, Virchow's Arch. 6 (1854) 562.

[2] M. Buchanan, J. Arrault, M.E. Cates, Langumir 14 (1998) 7371.

[3] M. Buchanan, S.U. Egelhaaf, M. Cates. Laugmuir 16, 3718 (2000).

[4] V. Frette, I. Tsafrir, M. Guedeau-Boudeville, L. Jullien, D. Kandel, J. Stavans, Phys. Rev. Lett. 83 (1999) 2465.

[5] I. Sakurai, Y. Kawamura, Biochim. Biophys. Acta (M) 735 (1984) 347.

[6] K. Mishima, K. Yoshiyama, Biochim. Biophys. Acta (M) 904 (1987) 149.

[7] E.M. Slayter, H.S. Slayter, Light and Electron Microscopy, Cambridge University Press, New York, 1992, pp. 168182.

[8] K. Bhadriraju, J. Bellare, A cryo-SEM technique developed and applied to surfactant liposomes, Proceedings of the 51st Annual Meeting of the Microscopy Society of America, Ohio, USA, 1993.

[9] R.C. Iyer, C. Manohar, J. Bellare, XXIV Annual Conference on Electron Microscopy and Allied Fields (EMSI), Chandigarh, 2001.

[10] R. Lipowsky, C. Heirgeist, J. Phys. II France 6 (1996) 1465.

[11] A. Suape, J. Colloid Interface Sci. 58 (3) (1977) 549.

[12] W. Helfrich, Z. Natureforsch 28c (1973) 693.

[13] G. Hota, Jayesh Bellare, Kartic C. Khilar, C. Manohar, Reactive organization of particles into macroscopic length scales by myelin growth, Curr. Sci. 80 (11) (2001) 14061407. 